\title{
RESEARCH
}

Open Access

\section{Time course of neuropathological events in hyperhomocysteinemic amyloid depositing mice reveals early neuroinflammatory changes that precede amyloid changes and cerebrovascular events}

Erica M. Weekman ${ }^{\dagger}$, Tiffany L. Sudduth ${ }^{\dagger}$, Brittani R. Price, Abigail E. Woolums, Danielle Hawthorne, Charles E. Seaks and Donna M. Wilcock* (1)

\begin{abstract}
Background: Vascular contributions to cognitive impairment and dementia (VCID) are the second leading cause of dementia behind only Alzheimer's disease (AD); however, VCID is commonly found as a co-morbidity with sporadic AD. We have previously established a mouse model of VCID by inducing hyperhomocysteinemia in both wild-type and amyloid depositing mice. While we have shown the time course of neuropathological events in the wild-type mice with hyperhomocysteinemia, the effect of amyloid deposition on this time course remains unknown; therefore, in this study, we determined the time course of neuropathological changes in our mouse model of hyperhomocysteinemia-induced VCID in amyloid depositing mice.
\end{abstract}

Methods: APP/PS1 mice were placed on either a diet deficient in folate and vitamins B6 and B12 and enriched in methionine to induce hyperhomocysteinemia or a control diet for 2, 6, 10, 14, or 18 weeks. Immunohistochemistry and gene expression analysis were used to determine neuroinflammatory changes. Microhemorrhages and amyloid deposition were analyzed using histology and, finally, behavior was assessed using the 2-day radial arm water maze.

Results: Neuroinflammation, specifically a pro-inflammatory phenotype, was the first pathological change to occur. Specifically, we see a significant increase in gene expression of tumor necrosis factor alpha, interleukin 1 beta, interleukin 6 , and interleukin 12 a by 6 weeks. This was followed by cognitive deficits starting at 10 weeks. Finally, there is a significant increase in the number of microhemorrhages at 14 weeks on diet as well as redistribution of amyloid from the parenchyma to the vasculature.

Conclusions: The time course of these pathologies points to neuroinflammation as the initial, key player in homocysteine-induced VCID co-morbid with amyloid deposition and provides a possible therapeutic target and time points.

Keywords: Alzheimer's disease, Vascular contributions to cognitive impairment and dementia, Hyperhomocysteinemia, Neuroinflammation, Astrocytic end-feet, Microhemorrhage

\footnotetext{
*Correspondence: donna.wilcock@uky.edu

${ }^{\dagger}$ Erica M. Weekman and Tiffany L. Sudduth contributed equally to this work.

Department of Physiology, Sanders-Brown Center on Aging, University of

Kentucky, 800 S. Limestone St., Lexington, KY 40536, USA
}

(c) The Author(s). 2019 Open Access This article is distributed under the terms of the Creative Commons Attribution 4.0 International License (http://creativecommons.org/licenses/by/4.0/), which permits unrestricted use, distribution, and reproduction in any medium, provided you give appropriate credit to the original author(s) and the source, provide a link to the Creative Commons license, and indicate if changes were made. The Creative Commons Public Domain Dedication waiver (http://creativecommons.org/publicdomain/zero/1.0/) applies to the data made available in this article, unless otherwise stated. 


\section{Background}

Vascular contributions to cognitive impairment and dementia (VCID) are increasingly recognized as a significant cause of dementia, behind only Alzheimer's disease (AD) [1]. Furthermore, VCID co-morbid with $\mathrm{AD}$ is extremely common, estimated to occur in at least $60 \%$ of $\mathrm{AD}$ cases [2]. Clinicalneuropathological correlation data has revealed that cerebrovascular pathologies increase the likelihood of dementia for a given amount of $\mathrm{AD}$ pathology. In early-onset familial $\mathrm{AD}$, cerebrovascular abnormalities as detected by neuroimaging appear to precede detectable AD pathology. Despite our increasing understanding of VCID as a contributing factor to clinical dementia, our appreciation for the mechanistic underpinnings of VCID, and also identification of potential therapeutic targets to treat VCID, has been lacking, in part due to a lack of relevant animal models.

One of the challenges of developing models for VCID is that VCID is an umbrella term for a variety of cerebrovascular pathologies including micro- and macro-infarcts, micro- and macro-hemorrhages, cerebral hypoperfusion, periventricular and deep white matter hyperintensities, and stroke. We have been working on a mouse model of VCID characterized by inflammation, microhemorrhages, and cerebral hypoperfusion. The model is induced through diet modification to elevate plasma homocysteine, a nonprotein forming amino acid (Hcy), resulting in hyperhomocysteinemia (HHcy). Over 20 years ago, HHcy (elevated plasma homocysteine) was identified as an independent risk factor for stroke and vascular disease [3]. HHcy is associated with confirmed VCID and AD cases [4] and is recognized as an important risk factor for AD [5]. HHcy is associated with accelerated hippocampal atrophy [4] and cognitive loss in AD patients [6]. The association with hippocampal atrophy appears to be independent of $A \beta$ pathology [7]. HHcy [8-11] and/or low B12 [12] and folate levels $[13,14]$ are also accompanied by white matter lesions, indicative of vascular damage. Genetic mutations in cystathionine $\beta$ synthase, a key enzyme in the homocysteine metabolism pathway, lead to HHcy and are recognized as a cause of stroke in children and young adults [15].

We have previously shown that induction of HHcy in wild-type (WT) mice leads to neuroinflammation, microhemorrhages., and cognitive impairment, producing a clinically relevant mouse model of VCID [16]. We have also shown that HHcy leads to astrocytic end foot disruption beginning between 6 and 10 weeks on diet [17]. In our co-morbidity model, APP/PS1 mice placed on the HHcy diet show a switch to a pro-inflammatory phenotype, microhemorrhages, additive cognitive deficits, and a redistribution of amyloid from the parenchyma to the vasculature [18]. While we have previously described the time course of neuropathological changes in our WT mice on the HHcy diet, the effect of amyloid deposition on this time course remains unknown. In this study, we examined neuroinflammation, microhemorrhages, amyloid deposition, and cognition along a time course of $2,6,10,14$, and 18 weeks in our comorbidity model. Similar to our WT HHcy model, neuroinflammation appears first at 6 weeks, followed by significant cognitive deficits at 10 weeks, and finally, microhemorrhages and redistribution of amyloid from the parenchyma to the vasculature occurred after 14 weeks on diet.

\section{Materials and methods}

\section{Animals}

Female and male APP/PS1 mice (C57BL/6 mice carrying human APPSwe and PS1-dE9 mutations) were bred in house and aged 6-8 months before starting a diet deficient in folate and vitamins B6 and B12 and enriched in methionine (Envigo TD130867; Envigo, Indianapolis, Indiana) or a control diet with normal levels of folate, vitamins B6 and B12, and methionine (Envigo TD01636; Envigo, Indianapolis, Indiana). Mice were on diet for 2, 6, 10, 14, or 18 weeks. Sample sizes, sex distribution, and homocysteine levels are summarized in Table 1. Homocysteine levels

Table 1 Sample size, sex distributions, and homocysteine levels

\begin{tabular}{|c|c|c|c|c|}
\hline \multirow{2}{*}{$\begin{array}{l}\text { Weeks on } \\
\text { diet } \\
\text { (HHcy or } \\
\text { Cont diet) }\end{array}$} & \multicolumn{4}{|c|}{ APP/PS1 } \\
\hline & $\bar{F}$ & Mean plasma Hcy ( $\mu \mathrm{mol} / \mathrm{L})$ & M & Mean plasma Hcy ( $\mu \mathrm{mol} / \mathrm{L})$ \\
\hline $2-\mathrm{HHcy}$ & 3 & $42.2 \pm 12.1$ & 2 & $39.5 \pm 10.1$ \\
\hline 2 -Cont & 3 & $6.2 \pm 0.8$ & 3 & $4.9 \pm 1.8$ \\
\hline 6- - H Hcy & 5 & $64.4 \pm 8.2$ & 5 & $59.3 \pm 9.8$ \\
\hline 6-Cont & 6 & $5.2 \pm 0.9$ & 6 & $5.5 \pm 0.8$ \\
\hline 10-HHcy & 4 & $69.3 \pm 6.2$ & 3 & $71.6 \pm 5.1$ \\
\hline 10 -Cont & 6 & $4.4 \pm 1.8$ & 6 & $6.6 \pm 1.2$ \\
\hline 14- HHcy & 5 & $88.6 \pm 14.1$ & 5 & $77.2 \pm 6.9$ \\
\hline 14-Cont & 7 & $5.6 \pm 1.4$ & 7 & $4.8 \pm 1.1$ \\
\hline 18- HHcy & 2 & $71.5 \pm 7.8$ & 5 & $64.3 \pm 8.4$ \\
\hline 18-Cont & 6 & $4.9 \pm 0.7$ & 6 & $5.7 \pm 0.5$ \\
\hline
\end{tabular}


were measured by the clinical laboratory at the University of Kentucky Clinic. At each time point, the APP/PS1 mice on the HHcy diet had significantly higher levels of homocysteine compared to mice on the control diet. This study was approved by the University of Kentucky Institutional Animal Care and Use Committee and conformed to the National Institutes of Health Guide for the Care and Use of Animals in Research.

\section{Behavior testing}

The 2-day radial arm water maze behavior test was performed the week prior to tissue harvesting at the University of Kentucky Rodent Behavior Core. The 20day behavior task was performed as previously described [19]. Briefly, a 6-arm maze was submerged in a pool of water with a goal platform placed at the end of one arm. Each mouse performed 15 trials over the 2 days and began each trial in a different arm while the goal platform remained the same. The number of errors (incorrect arm entries) was counted over a 60 -s period. Errors were averaged for three trials resulting in 10 blocks over the 2 days. Blocks 1-5 comprised day 1 trials while blocks 6-10 comprise day 2 trials.

\section{MRI}

Mice were imaged by $\mathrm{T} 2 *$ MRI the week prior to tissue harvesting. Mice were imaged with a 7-T Bruker ClinScan MRI system (Bruker, Billerica, MA) with an MRI CryoProbe, delivering 2.5 times the signal to noise of a standard room temperature radiofrequency coil, located at the Magnetic Resonance Imaging and Spectroscopy Center at the University of Kentucky. Fourteen coronal slices were acquired with a FLASH sequence with a TR $165 \mathrm{~ms}$, TE $15.3 \mathrm{~ms}, 25^{\circ}$ flip angle, $448 \times 448$ matrix, $0.4 \mathrm{~mm}$ thick, $20 \%$ gap, 0.033 $\mathrm{mm} \times 0.033 \mathrm{~mm}$ resolution, 10 averages, and TA 24 min. Mice were anesthetized with $1.3 \%$ isoflurane using an MRI compatible vaporizer. They were then positioned prone and held in place on the scanning bed using tooth and ear bars. The animals were maintained at $37^{\circ}$ with a water-heated scanning bed. Body temperature, heart, and respiration rates were monitored. T2* MRI images were analyzed by one blinded investigator who identified abnormalities that resembled hemorrhagic infarcts. These infarcts were counted, and this number was normalized to the number of images counted to provide a per section count.

\section{Tissue processing and histology}

After a lethal injection of Beuthanasia-D, blood was collected for plasma and the mice were perfused with 25 $\mathrm{mL}$ normal saline. Brains were rapidly removed and bisected along the mid-sagittal plane. The left half was immersion fixed in $4 \%$ paraformaldehyde for $24 \mathrm{~h}$ while the right half was dissected into the frontal cortex, posterior cortex, hippocampus, and the rest of brain and flash frozen in liquid nitrogen and then stored at $80{ }^{\circ} \mathrm{C}$. The left hemibrain was passed through $10 \%, 20 \%$, and finally $30 \%$ sucrose solutions for cryoprotection prior to sectioning. Using a sliding microtome, $25-\mu \mathrm{m}$ frozen horizontal sections were collected and stored free floating in $1 \times$ DPBS-containing sodium azide at $4{ }^{\circ} \mathrm{C}$.

Eight sections ( $25 \mu \mathrm{m}$ thick) spaced $600 \mu \mathrm{m}$ apart were selected for free floating immunohistochemistry for CD11b (rat monoclonal, 1:1000 dilution, AbD Serotec, Raleigh, NC for the 6-14-week time points; rat monoclonal, 1:1000 dilution, BioLegend, San Diego, CA, for the 18 -week time point). To reduce variability due to the use of two separate CD11b antibodies, we analyzed the data as a percent of the control mice for that specific time point (with the control mice averaging 100\%) rather than direct comparison of the percent areas occupied by positive immunostain. Immunohistochemistry was performed as previously described [20]. Stained sections were mounted, air dried overnight, dehydrated, and coverslipped in DPX (Electron Microscopy Sciences, Hatfield, PA). Analysis was performed by measuring the percent area occupied by positive immunostain using the Nikon Elements AR image analysis system (Nikon Instruments, Melville, NY) as described previously [21].

Eight sections spaced $600 \mu \mathrm{m}$ apart were selected and mounted on slides for Congo red and Prussian blue staining as previously described [22, 23]. Congo red analysis was performed using the Zeiss Axio Scan.Z1 Slide Scanner (Carl Zeiss Microscopy, Jena, Germany) and the Nikon Elements AR image analysis system. Neutral red was used as a counterstain for Prussian blue. For analysis of microhemorrhages, Prussian blue-positive profiles across the entire hemibrain section were counted by a blinded individual and calculated as an average per section.

\section{$A \beta$ ELISA}

Biochemical analysis of $A \beta$ levels was performed as previously described [18]. Briefly, soluble protein was extracted from the right frontal cortex using PBS with complete protease and phosphatase inhibitor (Pierce Biotechnology). After centrifugation, the supernatant was labeled the "soluble" extract, and the pellet was homogenized in $70 \%$ formic acid. After centrifugation and neutralization with $1 \mathrm{M}$ Tris- $\mathrm{HCl}$, the supernatant was labeled the "insoluble" extract. Protein concentrations were determined using the BCA protein assay kit (Pierce Biotechnology). The Meso-Scale Discovery multiplex ELISA system was used to measure $A \beta 1-38, A \beta 1-40$, and $A \beta 1-42$ levels in the soluble and insoluble extracts (V-PLEX A $\beta$ Peptide Panel 1 (6E10) Kit; MSD). 


\section{Quantitative real-time RT-PCR}

RNA was extracted from the right hippocampus using the E.Z.N.A. total RNA kit (Omega-Bio-Tek, Norcross, GA) according to the manufacturer's instructions. RNA was quantified using the Biospec nano spectrophotometer (Shimadzu, Japan) and reverse transcribed to cDNA using the cDNA High Capacity kit (Thermo Fisher, Grand Island, NY) according to the manufacturer's instructions. RT-PCR was performed using the Fast TaqMan Gene Expression assay (Thermo Fisher). In each well of a 96-well plate, $0.5 \mu \mathrm{L}$ cDNA (100 ng, based on RNA concentrations) was diluted with $6.5 \mu \mathrm{L}$ RNase-free water. One microliter of the appropriate gene probe was added with $10 \mu \mathrm{L}$ of Fast TaqMan to each well. Target amplification was performed using the ViiA7 (Applied Biosystems, Grand Island, NY). All genes were normalized to $18 \mathrm{~S}$ rRNA and the fold change was determined using the $-\Delta \Delta \mathrm{Ct}$ method [24]. Table 2 shows the genes tested along with their PMID and TaqMan ID.

\section{Data analysis}

Data are presented as mean \pm SEM. Statistical analysis was performed using the JMP statistical analysis software program (SAS Institute, Cary, NC). Radial arm water maze data were analyzed by repeated measures ANOVA. We also performed Student's $t$ test on individual block data. Histological, immunohistochemical, and biochemical data were analyzed by one-way ANOVA for each time point, as well as across time points. Statistical significance was assigned where the $P$ value was lower than 0.05 .

\section{Results}

Neuroinflammation is the first pathological abnormality that we observe in our APP/PS1-HHcy model. Immunohistochemistry for CD11b, which stains for both activated and resting microglia, showed a similar trend as the WT mice on the HHcy diet (previously published [17]). By 6 weeks on diet, the APP/PS1 mice on the HHcy diet have significantly more CD11b staining compared to APP/PS1 mice on the control diet (Fig. 1a, b, i). This increase in staining is sustained at 10 and 14 weeks on the HHcy diet but is reduced to control levels by 18 weeks (Fig. 1c-i).

Table 2 Genes for RT-PCR

\begin{tabular}{lll}
\hline Gene of interest & PMID & TaqMan ID \\
\hline TNFa & NM_013693.3 & Mm.1293 \\
IL1 $\beta$ & NM_008361.3 & Mm.222830 \\
IL12a & NM_008351.2 & Mm.103783 \\
IL-6 & NM_031168.1 & Mm.1019 \\
IL10 & NM_010548.2 & Mm.874 \\
YM1 & NM_009892.2 & Mm.387173 \\
\hline
\end{tabular}

While immunohistochemistry for $\mathrm{CD} 11 \mathrm{~b}$ showed an increase in microglial staining, we were interested in the specific cytokines expressed. The pro-inflammatory markers TNF $\alpha$ and IL-1 $\beta$ were significantly increased in the APP/PS1 mice on the HHcy diet starting at 6 weeks on diet and remained elevated through 18 weeks on diet (Fig. 2a). IL-12a and IL-6, anther two pro-inflammatory markers, were significantly elevated at 6,10 , and 14 weeks but returned to control levels by 18 weeks on the HHcy diet (Fig. 2a). The anti-inflammatory marker IL10 was significantly elevated after 6 weeks on the HHcy diet but decreased by 10 weeks and was significantly decreased compared to controls by 18 weeks (Fig. 2b). Another anti-inflammatory marker, YM1, was significantly decreased at 6 and 14 weeks on the HHcy diet (Fig. 2b). Similar to the CD11b staining, the increases in proinflammatory cytokines starting at 6 weeks were also seen in the WT mice on the HHcy diet [17].

We tested spatial memory changes in the APP/PS1 mice on the HHcy and control diets using the 2-day radial arm water maze. After 6 weeks on the HHcy diet, the APP/PS1 mice were not significantly cognitively impaired compared to the APP/PS1 mice on control diet (Fig. 3a). By 10 weeks on diet, the APP/PS1 mice on the HHcy diet made significantly more errors only in the last block of trials on the second day (Fig. 3b), similar to WT mice on the HHcy diet for 10 weeks [17]. The APP/PS1 mice on the HHcy diet made significantly more errors on the last three blocks on the second day at 14 weeks compared to the APP/PS1 mice on control diet (Fig. 3c). After 18 weeks on either the HHcy or the control diet, both groups of mice made a similar number of errors and did not learn the task at all (Fig. 3d). This does not mean that one group was more impaired than the other; the mice have reached a ceiling effect with this particular behavior task and further cognitive decline cannot be seen with this task. However, neither group showed any learning and are therefore still considered impaired.

Using both T2* MRI and Prussian blue staining, we determined the time course of microhemorrhages due to the HHcy diet in our co-morbidity model. Both methods showed a significant increase in the number of microhemorrhages in the APP/PS1 mice beginning at 14 weeks on the HHcy diet compared to the APP/PS1 mice on the control diet (Fig. 4a-c). Again, in the WT mice on the HHcy diet, we see a significant increase in microhemorrhages at 14 weeks [17].

To determine brain beta-amyloid (A $\beta$ ) load, we performed biochemical assessment of soluble and insoluble $A \beta_{1-38}, A \beta_{1-40}$, and $A \beta_{1-42}$ in the Meso-Scale Discoveries V-Plex $A \beta$ assay. We found no significant effect of HHcy induction in $A \beta$ levels at any of the time points examined (Table 3). To determine changes in amyloid distribution, we performed Congo red staining, which 


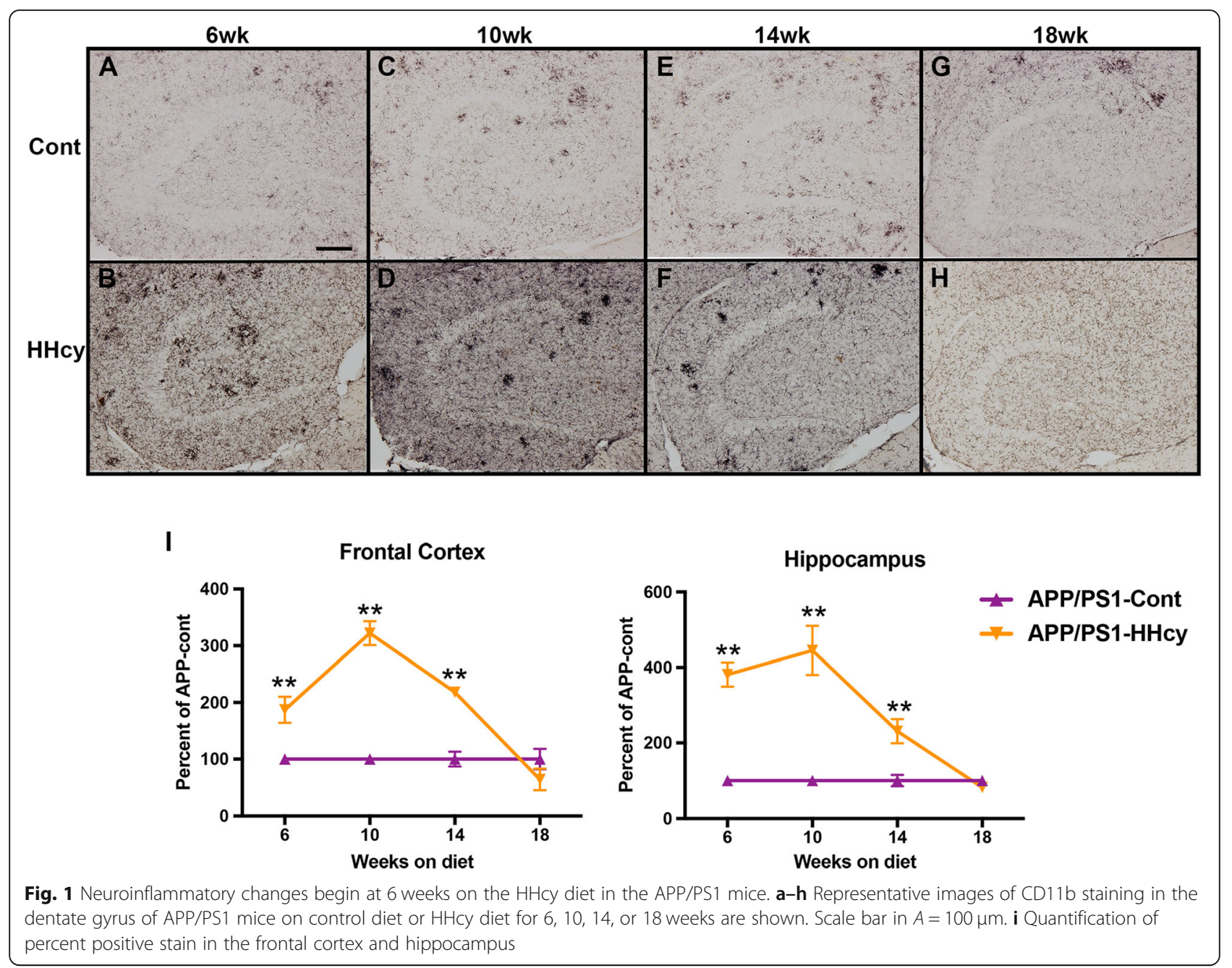

enables differentiation of vascular and parenchymal amyloid. At each time point, HHcy did not alter total levels of Congo red staining in both the frontal cortex and hippocampus (Fig. 5g, h). However, when parenchymal plaques and vascular amyloid are separated from total Congo red staining, it is revealed that HHcy leads to a redistribution of amyloid towards the vasculature (Fig. 5g, h). This redistribution occurs at 14 weeks on diet and continues to 18 weeks on diet (Fig. $5 \mathrm{c}-\mathrm{f}$ ).

\section{Discussion}

VCID is the second most common form of dementia yet it is commonly seen as a co-morbidity with AD [25-28]. While VCID is being increasingly recognized in the clinic, there are still a limited number of animal models to study mechanisms of VCID alone and when it is comorbid with AD. Previously, we characterized a mouse model of VCID and a co-morbidity model by inducing HHcy in WT and APP/PS1 mice, respectively [16-18]. We have shown the time course of neuropathological events in our VCID model alone [17]; however, we do not know the effect of amyloid deposition on this time course. Understanding and comparing the time course of these pathological changes could provide critical information for the mechanism of VCID alone and when it is co-morbid with AD. In this study, we show that neuroinflammation is the first pathology to occur, followed by cognitive changes and finally microhemorrhages and amyloid redistribution.

While little is known about inflammation's role in VCID, it is hypothesized that inflammation and oxidative stress play a key role in neurovascular dysfunction leading to cognitive decline. Chronic hypertension, a risk factor for VCID, leads to lumen narrowing and ultimately reduced blood flow. This decrease in blood flow induces expression of hypoxia inducible factor $1 \alpha$ which recruits macrophages from the systemic circulation as well as activation of endogenous microglia [29]. Activation of microglia leads to increases in proteases and free radicals that are released during remodeling of damaged vessels. Free radicals and proteases can break down the 

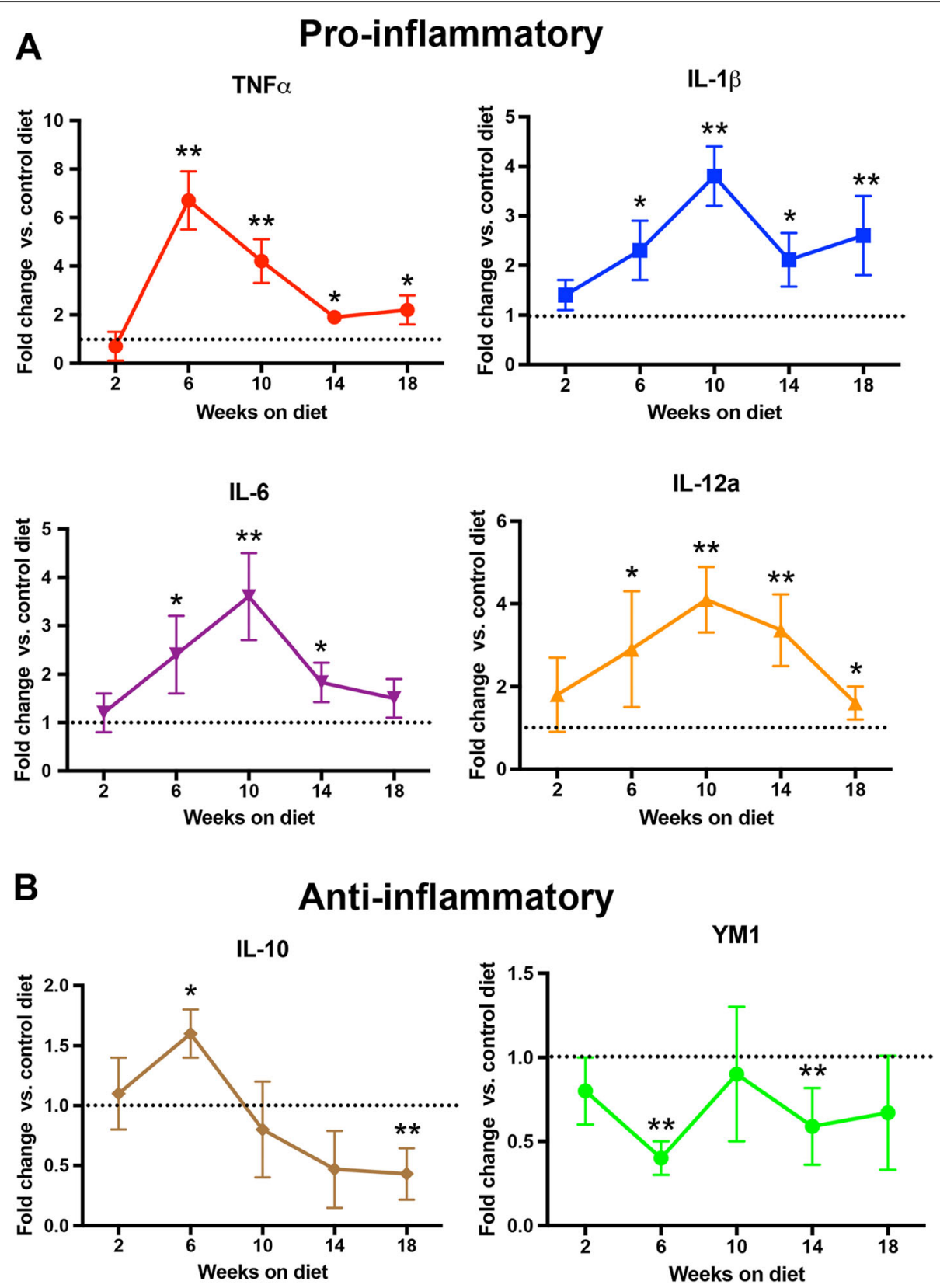

Fig. 2 Pro-inflammatory markers are increased at 6 weeks on the HHcy diet. Relative gene expression for pro- (a) and anti- (b) inflammatory markers. Data are presented as fold change from APP/PS1 mice on control diet at that time point. ${ }^{*}$ indicates $P<0.05,{ }^{* *}$ indicates $P<0.01$ compared to APP/PS1 mice on control diet for that time point

fibrotic basal lamina, disrupt tight junctions leading to vasogenic edema, and attack the myelinated fibers leading to demyelination [30, 31]. This increase in reactive oxygen species can also stimulate inflammatory pathways via toll-like receptors and lead to blood brain barrier breakdown. This vascular damage via inflammation most likely interferes with neurovascular coupling and the proliferation, migration, and differentiation of oligodendrocytes contributing to white matter damage and VCID [32]. In both our
VCID and co-morbidity model, alterations in the inflammatory phenotype are the first pathology to appear along with an increase in microglial staining. In addition, both models show a significant increase in several pro-inflammatory markers starting at 6 weeks before any disruptive vessel changes or cognitive decline is seen. While the microglial staining returned to control levels by 18 weeks on diet, the increase in pro-inflammatory markers remains throughout the course of the diet, possibly continuing to contribute 


\section{APP/PS1-Control $\neq$ APP/PS1-HHcy}
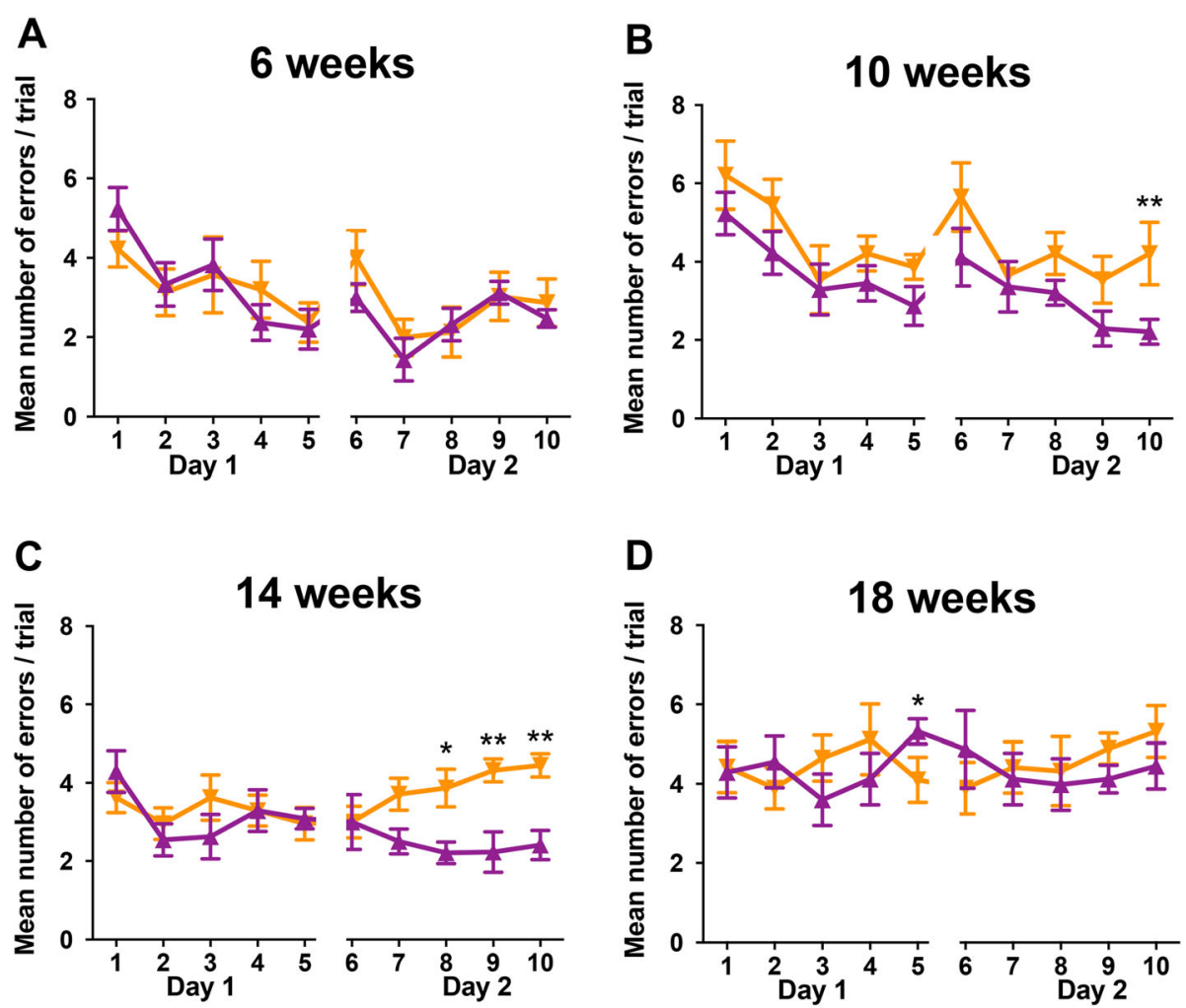

Fig. 3 Spatial memory deficits are seen starting at 10 weeks on the HHcy diet in APP/PS1 mice. a-d Two-day radial arm water maze data is shown for APP/PS1 mice on diet for 6, 10, 14, or 18 weeks. The mean number of errors per trial was calculated for each block (each block is the average of three trials). ${ }^{*}$ indicates $P<0.05$ and ${ }^{* *}$ indicates $P<0.01$ by repeated measures ANOVA

to the neuropathological changes seen later. Future studies will look more closely at the morphological phenotype of the microglia to determine the number of activated vs inactive microglia. Together, this suggests neuroinflammation is an initial, key player in homocysteine-induced VCID.

As mentioned above, the proteases and free radicals released by activated microglia can induce blood brain barrier breakdown and leakage. Matrix metalloproteinase 9 (MMP9) is a key protease shown to be activated by pro-inflammatory cytokines (IL-1 $\beta$ and $\mathrm{TNF} \alpha$ ) and has been shown to degrade tight junction proteins leading to leaky vessels and eventually microhemorrhages [33-35]. We have previously shown a significant increase in MMP9 gene expression and protease activity in both our VCID and co-morbidity models, with gene expression of MMP9 being significantly elevated after 24 weeks on diet $[16,18,36]$. MMP9 gene expression is still significantly elevated in the co-morbidity mice after 24 weeks on diet, showing a continued expression even after inflammation is returned to control levels [37]. In the current study, we show a slight increase in microhemorrhages at 10 weeks, with a significant increase by 14 weeks on diet. The appearance in microhemorrhages also coincides with the cognitive changes as well in both our VCID and co-morbidity models. At 10 weeks, there is a slight decline in cognition that becomes more pronounced by 14 weeks. With neuroinflammatory changes occurring before the appearance of microhemorrhages, we hypothesize that neuroinflammation induces MMP9 activation leading to microhemorrhages and then cognitive decline.

Another target of MMP9 includes the dystroglycans, specifically $\beta$-dystroglycan, which forms a complex that anchors the astrocytic end-foot to the basement membrane surrounding arterioles and capillaries $[38,39]$. As part of the neurovascular unit, astrocytic end-feet and their anchoring complexes function to maintain the ionic and osmotic homeostasis of the brain. Disruption of these homeostatic processes by breakdown of the anchoring complex and removal of the end-feet from the vasculature could lead to cognitive decline. Previously, we have shown a significant decrease in several astrocytic 


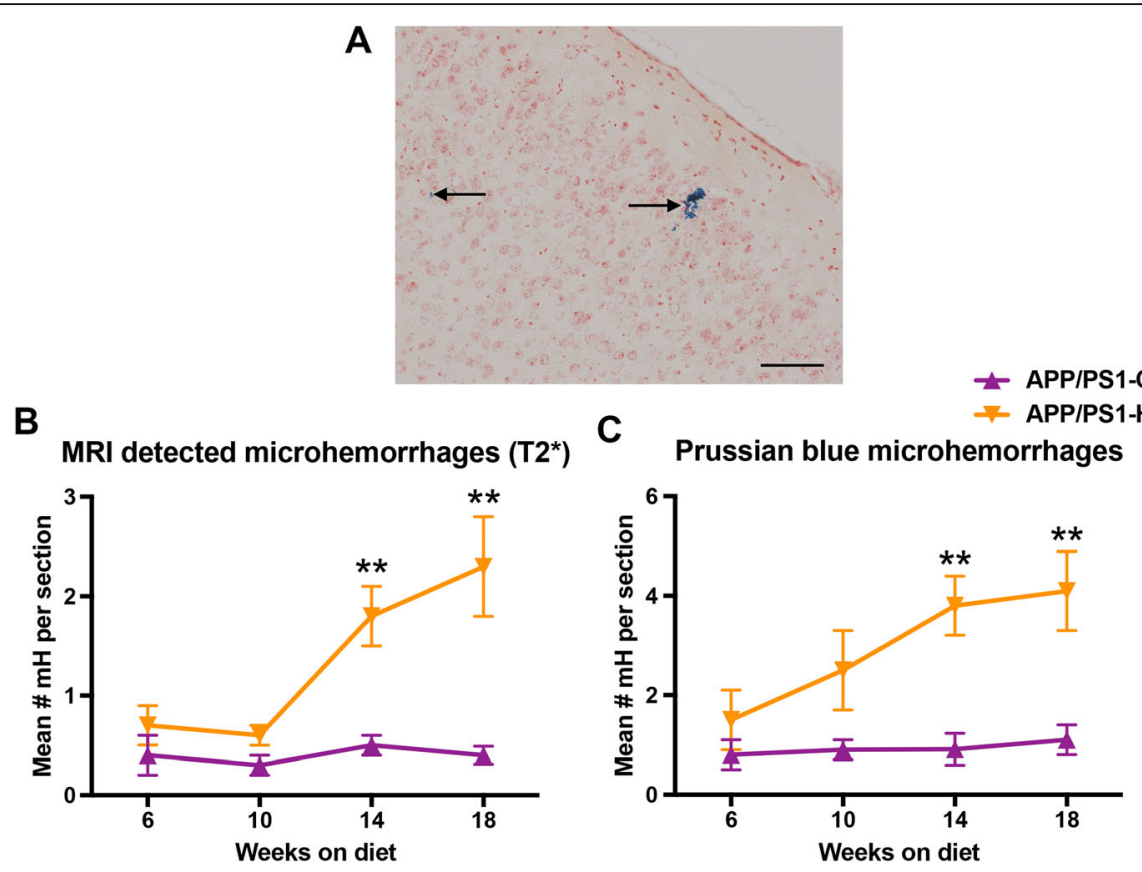

Fig. 4 Microhemorrhages increase at 14 weeks on the HHcy diet in APP/PS1 mice. a Representative image of Prussian blue-positive microhemorrhage in the frontal cortex. Magnification $=\times 200$. Scale bar $=50 \mu \mathrm{m}$, arrows indicate microhemorrhages. Quantification of the mean number of microhemorrhages per section from T2* MRI (b) or Prussian blue histology $(\mathbf{c}){ }^{* *}$ indicates $P<0.01$ compared to APP/PS1 mice on control diet for that time point

end-feet markers by 10 weeks on diet in our WT mice [17]. This coincides with the slight increase in microhemorrhages at 10 weeks as well as the slight behavioral deficits at 10 weeks seen in both our models. In addition, it has been shown that astrocytic end-foot disruption is associated with cerebral amyloid angiopathy in both $\mathrm{AD}$ and mouse models of cerebral amyloid angiopathy [20]. In our comorbidity model, since we have shown a redistribution of Congophilic amyloid from the parenchyma to the vasculature that occurs at 14 weeks on diet, we hypothesize that, similar to the WT HHcy mice, the co-morbidity mice will also have astrocyte end-feet degeneration around a similar time point. If so, with microhemorrhages and astrocytic end-foot disruption occurring before the redistribution of amyloid, astrocytes could play a key role in clearing amyloid through the vasculature. Any disruptions in the astrocytes could then lead to hindered clearance and thus the redistribution seen in our co-morbidity model.

\section{Conclusions}

Taken together, we have shown that neuroinflammation, specifically, an elevation of pro-inflammatory cytokines, is the initial change in both our VCID and co-morbidity mouse models and that the presence of amyloid does not alter the time course of

Table 3 Biochemical A $\beta$ assessment

\begin{tabular}{|c|c|c|c|c|c|c|c|}
\hline \multirow[t]{2}{*}{ Group } & \multirow{2}{*}{$\begin{array}{l}\text { Time- } \\
\text { point } \\
\text { (weeks) }\end{array}$} & \multicolumn{2}{|l|}{$A \beta_{1-38}$} & \multicolumn{2}{|l|}{$A \beta_{1-40}$} & \multicolumn{2}{|l|}{$A \beta_{1-42}$} \\
\hline & & Soluble & Insoluble & Soluble & Insoluble & Soluble & Insoluble \\
\hline \multirow[t]{4}{*}{ Control } & 6 & $0.03 \pm 0.01$ & $12.2 \pm 2.8$ & $0.9 \pm 0.2$ & $410 \pm 95.2$ & $0.7 \pm 0.2$ & $619 \pm 87.2$ \\
\hline & 10 & $0.05 \pm 0.03$ & 20.64 .4 & $0.4 \pm 0.1$ & $550 \pm 87.6$ & $0.8 \pm 0.3$ & $670 \pm 69.5$ \\
\hline & 14 & $0.08 \pm 0.02$ & $24.8 \pm 6.8$ & $0.8 \pm 0.6$ & $668 \pm 100.1$ & $1.4 \pm 0.6$ & $1214 \pm 85$ \\
\hline & 18 & $0.12 \pm 0.06$ & $19.9 \pm 8.7$ & $1.5 \pm 0.4$ & $942 \pm 206$ & 1.20 .4 & $1513 \pm 97$ \\
\hline \multirow[t]{4}{*}{ HHcy } & 6 & $0.02 \pm 0.01$ & $13.2 \pm 1.3$ & $1.1 \pm 0.3$ & $570 \pm 120.2$ & $0.9 \pm 0.3$ & $555 \pm 98.7$ \\
\hline & 10 & $0.14 \pm 0.07$ & $29.6 \pm 9.4$ & $0.8 \pm 0.2$ & $654 \pm 126.4$ & $0.7 \pm 0.2$ & $741 \pm 59.7$ \\
\hline & 14 & $0.09 \pm 0.03$ & $22.5 \pm 7.6$ & $0.7 \pm 0.1$ & $781 \pm 154.2$ & $1.4 \pm 0.6$ & $1356 \pm 96$ \\
\hline & 18 & $0.16 \pm 0.08$ & $19.6 \pm 8.4$ & $0.6 \pm 0.2$ & $1150 \pm 215$ & $1.5 \pm 0.4$ & $1641 \pm 79$ \\
\hline
\end{tabular}




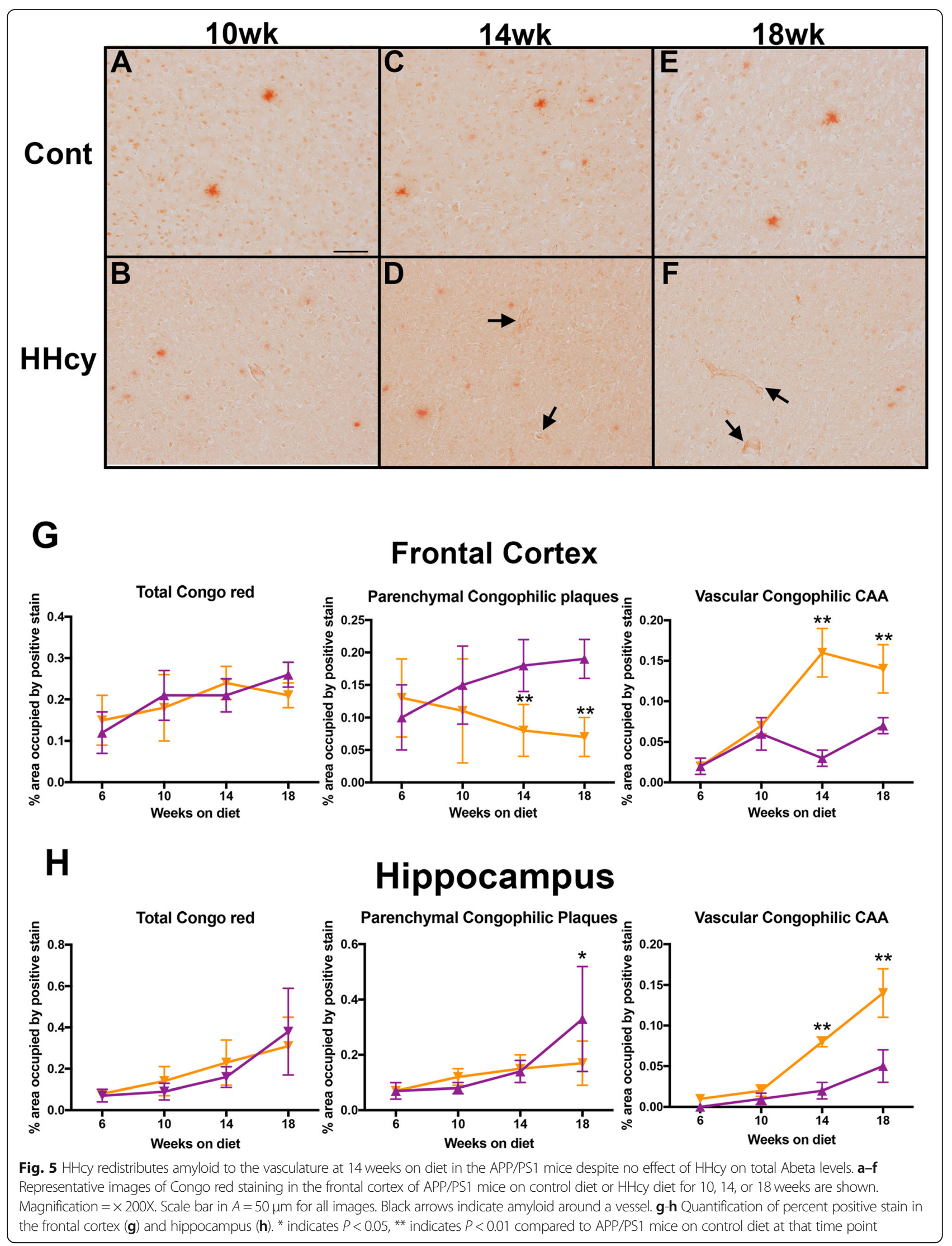


neuropathological events in our mouse model. Overall, this data provides several therapeutic targets and time points that can be tested in our mouse models as well as potential overlapping pathologies to look for in other VCID and AD models and human tissue.

\section{Abbreviations}

AD: Alzheimer's disease; Hcy: Homocysteine; HHcy: Hyperhomocysteinemia; IL10: Interleukin 10; IL12a: Interleukin 12 a; IL1ß: Interleukin 1 beta; IL6: Interleukin 6; MMP: Matrix metalloproteinase; TNFa: Tumor necrosis factor alpha; VCID: Vascular contributions to cognitive impairment and dementia; WT: Wild-type; YM1: Chitinase-like 3

\section{Acknowledgements}

Not applicable.

\section{Authors' contributions}

EMW and TLS performed the data collection and analysis, interpreted the data, and prepared the manuscript. BRP, AEW, DH, and CES assisted in the data collection. DMW conceived of the studies, analyzed and interpreted the data, and edited the final manuscript. All authors read and approved the final manuscript.

\section{Funding}

Research reported in this manuscript was funded by fellowship F31NS092202 (EMW), grants 1RO1NS079637 (DMW) and 1RO1NS097722 (DMW) from the National Institutes of Health, and training grant \#5T32GM118292 (CES) from the National Institute of General Medical Sciences. The content is solely the responsibility of the authors and does not necessarily represent the official views of the National Institutes of Health.

\section{Availability of data and materials}

Data sharing is not applicable to this article as no datasets were generated or analyzed during the current study.

Ethics approval and consent to participate

Not applicable.

\section{Consent for publication}

Not applicable.

\section{Competing interests}

The authors declare that they have no competing interests.

\section{Received: 9 May 2019 Accepted: 19 December 2019}

Published online: 30 December 2019

\section{References}

1. Levine DA, Langa KM. Vascular cognitive impairment: disease mechanisms and therapeutic implications. Neurotherapeutics. 2011;8(3):361-73.

2. Snyder HM, Corriveau RA, Craft S, Faber JE, Greenberg SM, Knopman D, et al. Vascular contributions to cognitive impairment and dementia including Alzheimer's disease. Alzheimers Dement. 2015;1 1(6):710-7.

3. Refsum H, Ueland PM, Nygard O, Vollset SE. Homocysteine and cardiovascular disease. Annu Rev Med. 1998;49:31-62.

4. Clarke R, Smith AD, Jobst KA, Refsum H, Sutton L, Ueland PM. Folate, vitamin B12, and serum total homocysteine levels in confirmed Alzheimer disease. Arch Neurol. 1998;55(11):1449-55.

5. Beydoun MA, Beydoun HA, Gamaldo AA, Teel A, Zonderman AB, Wang Y. Epidemiologic studies of modifiable factors associated with cognition and dementia: systematic review and meta-analysis. BMC Public Health. 2014;14: 643.

6. Oulhaj A, Refsum H, Beaumont H, Williams J, King E, Jacoby R, et al. Homocysteine as a predictor of cognitive decline in Alzheimer's disease. Int J Geriatr Psychiatry. 2010;25(1):82-90.

7. Choe YM, Sohn BK, Choi HJ, Byun MS, Seo EH, Han JY, et al. Association of homocysteine with hippocampal volume independent of cerebral amyloid and vascular burden. Neurobiol Aging. 2014;35(7):1519-25.
8. Hogervorst E, Ribeiro HM, Molyneux A, Budge M, Smith AD. Plasma homocysteine levels, cerebrovascular risk factors, and cerebral white matter changes (leukoaraiosis) in patients with Alzheimer disease. Arch Neurol. 2002;59(5):787-93.

9. Rajagopalan P, Hua X, Toga AW, Jack CR Jr, Weiner MW, Thompson PM. Homocysteine effects on brain volumes mapped in 732 elderly individuals. Neuroreport. 2011;22(8):391-5.

10. Kloppenborg RP, Geerlings MI, Visseren FL, Mali WP, Vermeulen M, van der Graaf $Y$, et al. Homocysteine and progression of generalized small-vessel disease: the SMART-MR study. Neurology. 2014;82(9):777-83.

11. Hooshmand B, Polvikoski T, Kivipelto M, Tanskanen M, Myllykangas L, Erkinjuntti T, et al. Plasma homocysteine, Alzheimer and cerebrovascular pathology: a population-based autopsy study. Brain. 2013;136(Pt 9):2707-16.

12. de Lau LM, Smith AD, Refsum H, Johnston C, Breteler MM. Plasma vitamin B12 status and cerebral white-matter lesions. J Neurol Neurosurg Psychiatry. 2009;80(2):149-57.

13. Iosifescu DV, Papakostas Gl, Lyoo IK, Lee HK, Renshaw PF, Alpert JE, et al. Brain MRI white matter hyperintensities and one-carbon cycle metabolism in non-geriatric outpatients with major depressive disorder (part I). Psychiatry Res. 2005;140(3):291-9.

14. Scott TM, Tucker KL, Bhadelia A, Benjamin B, Patz S, Bhadelia R, et al. Homocysteine and $B$ vitamins relate to brain volume and white-matter changes in geriatric patients with psychiatric disorders. Am J Geriatr Psychiatry. 2004;12(6):631-8.

15. Mudd SH, Finkelstein JD, Irreverre F, Laster L. Homocystinuria: an enzymatic defect. Science. 1964;143(3613):1443-5.

16. Sudduth TL, Powell DK, Smith CD, Greenstein A, Wilcock DM. Induction of hyperhomocysteinemia models vascular dementia by induction of cerebral microhemorrhages and neuroinflammation. J Cereb Blood Flow Metab. 2013;33(5):708-15.

17. Sudduth TL, Weekman EM, Price BR, Gooch JL, Woolums A, Norris CM, et al. Time-course of glial changes in the hyperhomocysteinemia model of vascular cognitive impairment and dementia (VCID). Neuroscience. 2017; 341:42-51.

18. Sudduth TL, Weekman EM, Brothers HM, Braun K, Wilcock DM. beta-amyloid deposition is shifted to the vasculature and memory impairment is exacerbated when hyperhomocysteinemia is induced in APP/PS1 transgenic mice. Alzheimers Res Ther. 2014;6(3):32.

19. Alamed J, Wilcock DM, Diamond DM, Gordon MN, Morgan D. Two-day radial-arm water maze learning and memory task; robust resolution of amyloid-related memory deficits in transgenic mice. Nat Protoc. 2006;1(4): $1671-9$.

20. Wilcock DM, Lewis MR, Van Nostrand WE, Davis J, Previti ML, Gharkholonarehe N, et al. Progression of amyloid pathology to Alzheimer's disease pathology in an amyloid precursor protein transgenic mouse model by removal of nitric oxide synthase 2. J Neurosci. 2008;28(7):1537-45.

21. Sudduth TL, Wilson JG, Everhart A, Colton CA, Wilcock DM. Lithium treatment of APPSWDI/NOS2-/- mice leads to reduced hyperphosphorylated tau, increased amyloid deposition and altered inflammatory phenotype. PLoS One. 2012;7(2):e31993.

22. Wilcock DM, Gordon MN, Morgan D. Quantification of cerebral amyloid angiopathy and parenchymal amyloid plaques with Congo red histochemical stain. Nat Protoc. 2006;1(3):1591-5.

23. Wilcock DM, Rojiani A, Rosenthal A, Subbarao S, Freeman MJ, Gordon MN, et al. Passive immunotherapy against Abeta in aged APP-transgenic mice reverses cognitive deficits and depletes parenchymal amyloid deposits in spite of increased vascular amyloid and microhemorrhage. J Neuroinflammation. 2004; 1 (1):24.

24. Livak KJ, Schmittgen TD. Analysis of relative gene expression data using real-time quantitative PCR and the 2(-Delta Delta C(T)) method. Methods. 2001;25(4):402-8.

25. Bowler JV, Munoz DG, Merskey H, Hachinski V. Fallacies in the pathological confirmation of the diagnosis of Alzheimer's disease. J Neurol Neurosurg Psychiatry. 1998;64(1):18-24.

26. James BD, Bennett DA, Boyle PA, Leurgans S, Schneider JA. Dementia from Alzheimer disease and mixed pathologies in the oldest old. JAMA. 2012; 307(17):1798-800.

27. Langa KM, Foster NL, Larson EB. Mixed dementia: emerging concepts and therapeutic implications. JAMA. 2004;292(23):2901-8.

28. Zekry D, Hauw JJ, Gold G. Mixed dementia: epidemiology, diagnosis, and treatment. J Am Geriatr Soc. 2002;50(8):1431-8. 
29. Semenza GL. Oxygen sensing, hypoxia-inducible factors, and disease pathophysiology. Annu Rev Pathol. 2014;9:47-71.

30. Cammer W, Bloom BR, Norton WT, Gordon S. Degradation of basic protein in myelin by neutral proteases secreted by stimulated macrophages: a possible mechanism of inflammatory demyelination. Proc Natl Acad Sci U S A. 1978;75(3):1554-8.

31. Rosenberg GA. Extracellular matrix inflammation in vascular cognitive impairment and dementia. Clin Sci (Lond). 2017;131(6):425-37.

32. Sim FJ, Zhao C, Penderis J, Franklin RJ. The age-related decrease in CNS remyelination efficiency is attributable to an impairment of both oligodendrocyte progenitor recruitment and differentiation. J Neurosci. 2002;22(7):2451-9.

33. Galis ZS, Muszynski M, Sukhova GK, Simon-Morrissey E, Unemori EN, Lark MW, et al. Cytokine-stimulated human vascular smooth muscle cells synthesize a complement of enzymes required for extracellular matrix digestion. Circ Res. 1994;75(1):181-9.

34. Vecil GG, Larsen PH, Corley SM, Herx LM, Besson A, Goodyer CG, et al. Interleukin-1 is a key regulator of matrix metalloproteinase-9 expression in human neurons in culture and following mouse brain trauma in vivo. J Neurosci Res. 2000;61(2):212-24.

35. Yang Y, Estrada EY, Thompson JF, Liu W, Rosenberg GA. Matrix metalloproteinase-mediated disruption of tight junction proteins in cerebral vessels is reversed by synthetic matrix metalloproteinase inhibitor in focal ischemia in rat. J Cereb Blood Flow Metab. 2007;27(4):697-709.

36. Sacharow SJ, Picker JD, Levy HL. In: Adam MP, Ardinger HH, Pagon RA, Wallace SE, LJH B, Stephens K, et al., editors. Homocystinuria caused by cystathionine beta-synthase deficiency. Seattle: GeneReviews((R)); 1993.

37. Weekman EM, Sudduth TL, Caverly CN, Kopper TJ, Phillips OW, Powell DK, et al. Reduced efficacy of anti-Abeta immunotherapy in a mouse model of amyloid deposition and vascular cognitive impairment comorbidity. J Neurosci. 2016;36(38):9896-907.

38. Michaluk P, Kolodziej L, Mioduszewska B, Wilczynski GM, Dzwonek J, Jaworski J, et al. Beta-dystroglycan as a target for MMP-9, in response to enhanced neuronal activity. J Biol Chem. 2007;282(22):16036-41.

39. Noell S, Wolburg-Buchholz K, Mack AF, Beedle AM, Satz JS, Campbell KP, et al. Evidence for a role of dystroglycan regulating the membrane architecture of astroglial endfeet. Eur J Neurosci. 2011;33(12):2179-86.

\section{Publisher's Note}

Springer Nature remains neutral with regard to jurisdictional claims in published maps and institutional affiliations.

Ready to submit your research? Choose BMC and benefit from:

- fast, convenient online submission

- thorough peer review by experienced researchers in your field

- rapid publication on acceptance

- support for research data, including large and complex data types

- gold Open Access which fosters wider collaboration and increased citations

- maximum visibility for your research: over $100 \mathrm{M}$ website views per year

At $\mathrm{BMC}$, research is always in progress.

Learn more biomedcentral.com/submissions 